\title{
Robert FOSSAERT
}

\author{
économiste
}

[Macro-sociologie et macro-économie]

(décembre 2009)

\section{"ESSAI SUR L'IGNORANCE.”}

Un document produit en version numérique par Jean-Marie Tremblay, bénévole, professeur de sociologie au Cégep de Chicoutimi

Courriel: jean-marie tremblay@uqac.ca

Site web pédagogique : http://www.uqac.ca/jmt-sociologue/

Dans le cadre de: "Les classiques des sciences sociales" Une bibliothèque numérique fondée et dirigée par Jean-Marie Tremblay, professeur de sociologie au Cégep de Chicoutimi Site web: http://classiques.uqac.ca/

Une collection développée en collaboration avec la Bibliothèque Paul-Émile-Boulet de l'Université du Québec à Chicoutimi

Site web: http://bibliotheque.uqac.ca/ 


\section{Politique d'utilisation de la bibliothèque des Classiques}

Toute reproduction et rediffusion de nos fichiers est interdite, même avec la mention de leur provenance, sans l'autorisation formelle, écrite, du fondateur des Classiques des sciences sociales, Jean-Marie Tremblay, sociologue.

Les fichiers des Classiques des sciences sociales ne peuvent sans autorisation formelle:

- être hébergés (en fichier ou page web, en totalité ou en partie) sur un serveur autre que celui des Classiques.

- servir de base de travail à un autre fichier modifié ensuite par tout autre moyen (couleur, police, mise en page, extraits, support, etc...),

Les fichiers (.html, .doc, .pdf., .rtf, .jpg, .gif) disponibles sur le site Les Classiques des sciences sociales sont la propriété des Classiques des sciences sociales, un organisme à but non lucratif composé exclusivement de bénévoles.

Ils sont disponibles pour une utilisation intellectuelle et personnelle et, en aucun cas, commerciale. Toute utilisation à des fins commerciales des fichiers sur ce site est strictement interdite et toute rediffusion est également strictement interdite.

L'accès à notre travail est libre et gratuit à tous les utilisateurs. C'est notre mission.

Jean-Marie Tremblay, sociologue

Fondateur et Président-directeur général, LES CLASSIQUES DES SCIENCES SOCIALES. 
Cette édition électronique a été réalisée par Jean-Marie Tremblay, bénévole, professeur de sociologie au Cégep de Chicoutimi à partir de :

\section{Robert Fossaert}

\section{“ESSAI SUR L'IGNORANCE.”}

Paris : texte inédit, décembre 2009, 7 pp.

[Autorisation formelle accordée par l'auteur le 20 juillet 2003 de diffuser ses oeuvres dans Les Classiques des sciences sociales. Le 25 février 2010, Monsieur Fossaert nous reconfirmait sa permission de diffuser cet opuscule.]

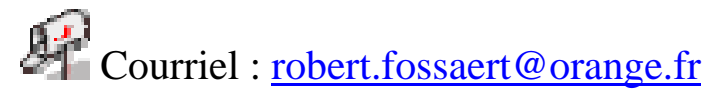

Polices de caractères utilisée :

Pour le texte: Times New Roman, 14 points.

Pour les citations : Times New Roman, 12 points.

Pour les notes de bas de page : Times New Roman, 12 points.

Édition électronique réalisée avec le traitement de textes Microsoft Word 2008 pour Macintosh.

Mise en page sur papier format : LETTRE (US letter), 8.5'’ x 11'’)

Édition numérique réalisée le 25 février 2010 à Chicoutimi, Ville de Saguenay, province de Québec, Canada.

\section{f Fait avec}


Robert FOSSAERT

\section{“ESSAI SUR L’IGNORANCE.”}

Paris : texte inédit, décembre 2009, 7 pp.

\section{Que sait-on et comment ?}

Avons-nous une idée claire de nos savoirs ? de ce qu'ils signifient et de ce qu'ils embrouillent ou qu'ils cachent? Avons-nous une petite idée de ce que nous ignorons ? Savons-nous tracer la frontière qui sépare nos savoirs effectifs de ce que d'autres semblent savoir ou de ce que la plupart des autres ignorent ? Savons-nous remplacer ces limites changeantes par une carte détaillée de ce qui figure, d'un pays ou d'un siècle à l'autre, des divers côtés de ces bornages qui ne sont certainement pas linéaires, ni stables dans l'espace et dans la durée ?

Pour simplifier un peu cet immense fouillis de questions, je vais faire masse de tous les humains. J'ai donc en vue les bientôt 9 milliards d'individus de l'espèce animale-humaine qui peuplent notre commune planète et je n'oublierai pas tout à fait la centaine de milliards d'humains des générations précédentes. Je dis la centaine, car mon ami Michel Louis Lévy, éminent démographe, avait actualisé en 1988 l'évaluation rétrospective de cette espèce, depuis son émergence du monde des primates. ${ }^{1}$ Il comptait, alors, quelques 80 milliards d'humains, chiffre que j'abonde et arrondis pour tenir compte de l'éxubérance démographique des décennies suivantes et de l'aléatoire des évaluations les plus anciennes. Toujours est-il que nos savoirs proviennent de ces cohortes d'ancêtres. Leur source première a été, tout au long des millénaires, l'activité banale d'animaux humains qui se

1 INED - Population et Société - Lettre n 224 : Combien est-il né d'hommes? 
sont peu à peu socialisés de façons diverses. Ils ont peuplé presque toute la planète, ont tiré leur nourriture des espèces animales et végétales là-présentes, ont appris à chasser, pêcher, cultiver la terre, domestiquer des animaux, s'abriter du froid et du soleil comme des intempéries, etc. Pendant ces milliers de millénaires, toujours laborieux, ils ont survécu aux aléas climatiques et aux tumultes naturels. Ils ont organisé leurs groupes, en coexistence comme au combat. Ils ont inventé des savoir-faire affinant les façons de l'agriculture, de l'élevage, de la navigation et de tout ce qui s'ensuit, jusqu'à esquisser des spécialisations dotées d'outils propres (armes, poterie, tissage, habitat, etc..).

Au fil des millénaires occupés de la sorte, la communication entre humains s'est enrichie. A force de corriger leurs éructations encore animales, les groupes étalés ou disjoints ont inventé de multiples langages. De lentes mutations ont mûri dans les plus substantiels de ces groupes ou sur les sites les plus féconds, dans des écarts naturellement protégés ou chez les initiateurs d'abris prolongeant la disponibilité des ressources tirées de la nature et en toutes autres occurrences favorables, y compris celles de la rapine. Les savoir-faire lentement élaborés, ont été diversement protégés et partagés. Les savoir-dire se sont condensés en récits, en rituels, en cérémonies, etc. Des théories c'est-à-dire des cortèges - de dires et d'actes ont été transmis d'une génération ou d'un groupe à l'autre. Des arts et métiers ont pris forme.

La taille et le style des groupes de convivance n'ont cessé d'être modifiés par une adversité naturelle aléatoire et par les spasmes contrastés des aléas intergroupaux dûs aux crises internes, aux rivalités de voisinage ou de rencontre et, de temps à autre, aux courants migratoires déclenchés par des famines, des rapines, des épidémies et d'autres secousses se propageant en cascade. L'échange et le combat ainsi entremêlés ont modifé, par crises ou par évolutions lentes, les us et coutumes propres à chaque groupe ou à chaque zone riche de groupes apparentés de quelque manière. Le guerrier et le marchand, mais aussi l'artisan faiseur d'objets échangeables ont enrichi la gamme des arts et métiers, bien au delà des travaux tirant du donné naturel les moyens de la survivance groupale. Précoce ou tardive, spontanée ou 
venue d'ailleurs, fragile ou diversement affermie, cette division du travail a débordé des rôles assignés aux genres et aux âges, pour s'enrichir de hiérarchies intra- et intergroupales.

Tous ces mouvements, infiniment variés et sans cesse défaits, repris et compliqués, ont été scandés par les détenteurs de pouvoirs, de savoirs ou d'autres charmes dont les opérations ont lentement enrichi les coutumiers de la vie quotidienne. La transmission de chaque coutumier a toujours été l'affaire de tous, à commencer par les mères. Souvent, ce travail collectif et répétitif, apprécié des vieillards et surveillé par les puissants, s'est cristallisé en spécialisations reconnues parmi lesquelles les soignants, les enseignants, les artistes et les savants d'aujourd'hui finissent par découvrir leur lointaine ascendance sous les allures des chamans, sorciers, mystagogues et autres sages que des ethnologues d'hier et d'aujourd'hui offrent comme repères. Les professionnels des choses distinctes des banalités naturelles ont ainsi pris des tournures fort diverses en se spécialisant. Ils ont inventé des façons de dire et de faire qui leur ont permis de garder mémoire de leurs activités et d'y initier d'autres humains.

Il faut lire les lignes précédentes avec respect pour tous les découvreurs et passeurs de savoirs nouveaux, tout en sachant que leur multitude demeurera à jamais inconnue. En effet, les états de choses d'avant l'écriture ne sont connaissables qu'au moyen de repères rares et indirects autant qu'incertains : des récits ultérieurs, des restes matériels aléatoires, d'occasionnelles grottes, tombes et autres conservateurs de traces éventuellement interprétables, etc. La documentation sur les milliers de millénaires d'avant l'écriture est un travail à jamais inachevé. Elle ne s'est enrichie peu à peu et non sans errements que par les créations et transmissions des diverses écritures qui ont pu être déchiffrées - pour la plupart, mais non toutes. Ces écritures fort variées ont laissé des traces en diverses régions du monde actuel, des hiéroglyphes égyptiens aux caractères chinois, aux alphabets phéniciens et aux ficelles mayas, exemples fort incomplets d'un énorme processus qui s'est étalé sur quelques petits millénaires, avant de contaminer le monde humain presque en son entier. 
Du fait de cette transformation lente mais radicale, la transmission des savoirs a été bouleversée du tout au tout. Le mouvement a été régionalement et socialement fort inégal. Régionalement, car il s'est opéré par plaques de contact ou par incursions de navigateurs ou de caravaniers, venus d'ailleurs lointains. Socialement, parce que les écritures, abruptes ou peu à peu raffinées, ont souvent et longtemps servi les puissants, qu'ils soient princes ou/et prêtres. Néanmoins, pour qui la considére dans toute sa généralité, la novation scripturale a foisonné de plus en plus. Elle a donné corps littéraire à des récits comme à des comptes, à des mythes et à des mystères, etc. Elle a fait éclore des métiers nouveaux pour les écrivants. Elle a été enrichie par des outils pour écrire, pour décrire, pour compter, pour observer, pour garder trace, etc. Elle a donné vie à des objets porteurs de choses immatérielles comme des pensées, ou irréelles comme des récits portant sur des ailleurs imaginaires, etc. Du plus archaïque des calendriers aux plus sophistiqués des véhicules et des vecteurs entrés dans l'usage, elle a permis l'éclosion de savoirs et de mesures de toutes sortes. Des œuvres de plus en plus distinctes des labeurs premiers de l'humanité ont enrichi sans cesse la gamme des allures et des postures humaines, c'est-à-dire des savoirs incorporés chez des hommes, mais de façon inégale et différentielle.

Dans ce nouveau monde aux perspectives infinies, la pensée humaine, parfois encore secrète dans certains de ses replis, s'est cependant déployée vers des masses immenses d'initiés. Elle a permis de faire école, au point que l'illettrisme est devenu autant que la misère un indicateur de sous-développement, pour une humanité qui tient le savoir comme une richesse à faire croître. De longue date, dans ses écoles les plus subtiles, elle a fait, de la pensée elle-même, un objet auquel penser, pour la mieux comprendre et la perfectionner. Cette philosophie aux habits si divers a permis de ressaisir maints savoirs établis, de réexaminer maints outils confirmés par un usage parfois immémorial, de compiler maintes interrogations et maintes quêtes et enquêtes, jusqu'à faire fleurir des sciences et des techniques diversi- 
fiables à l'infini, non sans les baptiser de noms sans cesse précisés, subdivisés et catalogués.

Des scribes en tous genres ont démultiplié les traces des savoirs préétablis, pour les répandre ou les enseigner. À ces fins, des matériaux durables ont succédé aux pierres taillées et aux tablettes gravées. Ces parchemins, ces roseaux encollés et tous les autres supports de l'écrit (en toutes ses déclinaisons) ont commencé de s'empiler dans des bibliothèques. Le papier a finalement triomphé un peu partout, suivi par les medias les plus divers. De fil en aiguille, quand les outils ont été machinés d'une production à l'autre, l'écriture a été emportée dans le même mouvement. Les machines à écrire, à calculer, à dessiner ont conflué jusqu'à rejoindre les torrents multimedias qui débordent en ce 21è siècle, sur la planète entière.

Ainsi se matérialise une nouvelle croûte terrestre, vaste brume d'images, énorme brouhaha de sons, infini empilement de savoirs qui enserre toute la planète et qui nappe la croûte naturelle sur laquelle les humains s'activent.

\section{Qu' ignore-t-on et pourquoi ?}

La masse de savoirs et d'ignorances qui encroûte désormais la Terre est un magma de coutumes, de paroles et d'écrits, d'images et de sons, d'actes et de mémoires incorporés dans toutes les pratiques de la vie humaine-sociale et, pour partie, tardivement empilés dans les "thèques" de toutes sortes. En parcourant la gamme complète des sociétés pour gravir l'échelle des modes d'initiation, d'apprentissage, de scolarisation et d'autres acculturations jusqu'à atteindre les fines pointes des sciences en marche et des philosophies en pleine inventivité, on ne quitte pas ce magma. Le dosage des savoirs et des ignorances varie certes d'un pays à l'autre, mais, même dans les laboratoires savants, les sachants les plus avisés sont eux aussi porteurs des ignoran- 
ces dont leur histoire personnelle les a garnis, leur vie durant, et que la quotidienneté banale entretient inévitablement.

Cette ignorance banale ne doit pas être confondue avec l'ignorance savante qui est celle de tous les véritables chercheurs, en toute société et en toute époque. Qu'ils orientent leur quête de manière fructueuse ou non, ces virtuoses du savoir-faire, ces explorateurs de territoires non ou mal connus, ces praticiens de l'expérimentation, ces aventuriers de l'idée neuve savent qu'ils n'en savent pas assez et cherchent à en savoir plus et mieux. Leurs tâtonnements, leurs expérimentations, leurs incursions s'accompagnent de questionnements et d'essais où des savoirs nouveaux mitonnent ou fulgurent jusqu'à trouver leur consécration dans les dires et pratiques de groupes locaux ou professionnels. L'imitation, hasardeuse ou stimulée, amorce la diffusion de ces savoirs neufs. Ailleurs et à d'autres moments, d'autres quêtes, diversement motivées, font surgir des savoirs analogues ou créent des savoirs supérieurs.

Ainsi le contraste entre l'ignorance et le savoir n'a pas la robuste simplicité de l'alternance du jour et de la nuit. Pour qui peut l'observer dans sa durée historique sur un site précis, il est formé de contorsions diverses et il est également coupé de syncopes que, parfois, aucune renaissance ne vient achever. Qui connaîtra la pleine sagesse de toutes les Babylone enfouies sous les sables du temps ? D'autant qu'entretenir l'ignorance est aussi une pratique sociale dont on trouve les traces en maintes sociétés anciennes et dont les résurgences modernes sont légion. Les sorciers de toute sorte qui tirent leur fascination d'un petit savoir ésotérique ont pour successeurs souvent efficaces des prophètes, parfois relayés par des institutions, maîtresses de secrets bien gardés. Par exemple, des savoirs astronomiques ou météorologiques garantissent les capacités miraculeuses de leurs opérateurs. Le soin des malades, la fragilité des récoltes, les monstruosités des élevages, les ruses et cruautés des guerrres, offrent de multiples occasions d'abus semblables. Les savoirs des puisatiers, des marins, des mineurs et des artisans de toutes variétés sont plus difficiles à convertir de la sorte, mais s'adornent aisément d'excroissances imaginaires. 
Quand vient l'heure des techniques élaborées, des productions machinées, des marchands inventeurs de commerces lointains, des finances au long cours et à long terme, etc, les savoirs correspondants s'offrent eux aussi à des manipulations toujours plus sophistiquées. Les fraudes, les escroqueries, les contrefaçons, les "délits d'initiés" débordent de l'ordinaire des roueries marchandes, des jeux publicitaires, des mensonges politiques, des propagandes fallacieuses, etc. à l'infini, pour tirer parti d'ignorances soigneusement entretenues.

Les sciences qui visent à la connaissance des objets naturels affermissent peu à peu leurs savoirs, à mesure que des outils convenables élargissent leur emprise. Des millénaires ont été nécessaires pour construire une idée claire du temps et de l'espace, à grand renfort d'horloges et de cartes, mais l'exploration extra-terrestre par satellites a relativisé ces acquis millénaires en ouvrant de facto une problématique relativiste, que l'expérimentation sub-atomique complique par ailleurs. Ensuite, sur les chemins battus et rebattus des chronologies affermies par des marqueurs tel le carbone 14, l'espace-temps qui se concrétise en parcours et en durées demeure une mine de savoirs et un réservoir d'ignorances non résorbées qui ne seront dévoilées qu'au bénéfice d'explorations expérimentales et théoriques nouvelles.

Les volcans mythifiés en Enfer ont tardé à perdre cette vertu même quand le magma naturel enrobé par la croûte terrestre (la réelle et non l'idéelle) est devenu, sous les assauts infinis de sciences foisonnantes, une part intelligible d'un univers matériel à jamais agité de mouvements qu'aucun "grand horloger" ne vient remonter. Mais il ne suffit pas que la tectonique des plaques terrestres ait commencé d'être repérée, pour que tous les tumultes sismiques deviennent compréhensibles, puis prévisibles ou que "l'infiniment lointain" de la matière devienne explorable. Celles des sciences naturelles qui s'appesantissent sur "l'infiniment petit" se libèrent des théologies productrices de révélations et de divinités, pour briser les atomes supposés insécables, mais elles ajoutent à l'ignorance un big bang "originel" dont les créationnistes font leurs choux gras. Et ainsi de suite : l'ignorance est le trésor de la recherche scientifique, le territoire inépuisable des savoirs à 
conquérir où chaque avancée donne naissance à des questions nouvelles en même temps qu'à des savoirs neufs.

\section{De la nature à la société...}

L'une des provinces les plus difficiles à conquérir concerne l'espèce humaine considérée dans sa matérialité animale. Si Darwin a fait révolution, c'est moins par la richesse des voies de recherche qu'il a reprises et élargies, que par la hauteur des obstacles idéologiques qu'il a aidé à surmonter. J'ai le sentiment que les changements en cours dans l'étude et la conception du cerveau humain (Changeux et al.) vont ouvrir de nouvelles brèches, à mesure que l'organisation neuronale de la pensée deviendra observable et intelligible. Les tumultes soulevés par les savoirs freudiens, relatifs à la sexualité dans ses rapports avec toutes les autres activités des humains, me semblent, en effet promis à des rebonds majeurs, dans la mesure où la physiologie bousculera peu à peu les hypothèses que les disciplines psychologiques ont rendu fécondes en ce domaine. Mais les recherches libérées par les Darwin et autres Freud ne réussiront jamais à dissiper les craintes et les brumes qui les cantonnent lorsqu'il s'agit de concevoir pleinement la matérialité de l'animal-humain. Ce disant, je ne vise pas principalement les ombres toujours renouvelées que des théologies obstinées font régner chez leurs fidèles, pour ce qui est des soins du corps, des normes de l'habillement, des usages alimentaires et de maints autres aspects des coutumes et des mœurs, car l'obstacle essentiel est beaucoup plus profond. Si profond qu'il n'y a aucun espoir de voir jamais l'humanité s'en dépêtrer totalement, quelles que soient les lumières que lui apporteront de nouveaux savoirs.

En effet, chaque individu de l'espèce animale-humaine est doté d'un cerveau qui le singularise à jamais. Sa pensée appréhende ce qu'elle peut saisir du monde extérieur à lui, mais elle est tout aussi forcément tournée vers elle-même. Elle est conscience de soi et elle devient conscience de sa finitude. Je note cela avec un vocabulaire 
certainement désuet, car je ne connais pas les multiples savoirs neufs, inéluctablement enrobés d'ignorances anciennes ou rajeunies, qui ont produit de nouvelles façons de dire que l'humain est mortel, que son être est transitoire et que la conscience de sa finitude s'entremêle avec sa sexualité. Néanmoins, Eros et Thanatos, ces mythes sans cesse revisités et reformulés en savoirs/ignorances dûment modernisés, ne perdent rien de leur pertinence. Aujourd'hui encore, le sujet, l'acteur, l'individu, le vécu et maintes autres figures de l'humain bouchent l'horizon du savoir.

Il faut sortir de ce piège pour bien comprendre le rapport entre le savoir et l'ignorance. Les individus de l'espèce humaine ont toujours expliqué le monde où ils vivent. Leur ignorance réelle a donné naissance à des divinités multiples, à des cultes durables, à des coutumes tirant leur évidence d'elles-mêmes et à une éternelle réactivation de mythes inexacts autant qu'immortels. En s'éloignant des autres espèces animales et en soumettant celles-ci, l'humanité a triomphé d'une partie des pesanteurs inhérentes à sa vie sur une planète qu'elle a faite sienne. Son efficacité croissante a permis de reconnaître l'objet dit nature sous des aspects de plus en plus nombreux. Mais elle peine encore à reconnaïtre l'objectivité de l'objet que l'on peut dire homme et si elle entrevoit de mieux en mieux l'objectivité de la société, elle n'en tarde pas moins à concevoir clairement l'objet qu'on peut nommer société.

J'abrège ces trois types d'objet, respectivement en $\mathbf{N}, \mathbf{H}$ ou $\mathbf{S}$. Il doit être bien entendu que l'objet $\mathbf{N}$ englobe les deux autres, tant il est vrai qu'il n'est sur Terre que des objets naturels, faits d'amas matériels que l'on dénomme aujourd'hui molécules, ondes ou d'autre manière pour désigner des choses plus vastes ou plus fines que mon ignorance m'interdit de cataloguer. L'objet $\mathbf{S}$ déborde de la nature, non point parce qu'il serait formé d'autres éléments, ni parce que l'espèce humaine se singulariserait par une origine "extra-terrestre", par une "création divine" ou par quelque autre propriété "surnaturelle", mais parce que la pensée ne se laisse capter que par d'autres êtres pensants. Au passage, je note que la communication qui peut s'établir entre des humains et des individus d'autres espèces animales n'est pas ici niée, même si 
son caractère bordurier n'est pas douteux. Et, par ailleurs, je m'abstiens de discuter, ici, des concrétions idéologiques évoquées par les termes dont j'ai fait usage ci-avant (extra-trerrestre, divin, surnaturel, etc.), car elles se réfèrent à des pratiques sociales bien vivantes (hélas !) dont l'appareillage relève des sciences visant l'objet $\mathbf{S}$.

En $\mathbf{H}$ comme en $\mathbf{S}$, les savoirs s'affermissent à mesure que leurs cibles peuvent être objectivées. Les savoir-faire se vérifient à l'épreuve du réel, les savoir-dire requièrent d'autres vérifications que l'humanité bricole ou codifie, mais en toute occurrence des précautions difficiles à concevoir et à appliquer commandent et contrôlent l'objectivation visée. Autrement dit les objets $\mathbf{H}$ et $\mathbf{S}$ ne sont jamais donnés - comme l'est l'objet $\mathbf{N}$ - ils sont à construire, si bien que leur échafaudage est toujours dans le champ des vérifications requises. L'ancrage au sol des peuplades puis des peuples de plus vaste envergure offre un puissant renfort à l'objectivation de l'objet $\mathbf{S}$ et contribue finalement à l'objectivation des individus de l'espèce $\mathbf{H}$. Mais les peuples sans feu ni lieu pour cause de nomadisme partagent néanmoins avec les sédentaires certains traits distinctifs tout aussi objectivants : le visible des us et coutumes (tels l'habillement, le langage, l'apparence physique, etc.). À partir de quoi, un énorme travail s'accomplit en $\underline{\mathrm{S}}$.

L'objectivation s'affirme quand s'accomplit le lent et tortueux parcours des chefferies jusqu'aux Etats de plus en plus détaillés. Alors, la longue litanie des villages et des villes et ports, des murs et chateaux, des édifices "sacrés", des chemins devenant routes, etc. imprime ses marques sur les territoires et leurs habitants. Et ainsi de suite, nonobstant les ruines et les destructions. De l'embryonnaire au plus développé des Etats, des appareils au fonctionnement de plus en plus abstrait et d'autres institutions plus souples se déploient sur des territoires fortement maillés où la richesse des relations sociales s'accroît d'autant plus que les liaisons offertes par les mers et les fleuves ou matérialisées par la route, puis le rail et tout ce qui s'ensuit entretissent leurs réseaux polyvalents, tandis que des medias de toute sorte prolifèrent bien au delà des limites étatiques que la haute mer n'a jamais respectées. La société devient un objet de plus en plus évident. 
Cependant, les individus, les familles et les autres mini-groupes de l'ordre $\mathbf{H}$ n'y acquièrent qu'une objectivation très indirecte et lacunaire. Les appareils des Etats travaillent plus que toutes les autres institutions sociales à rendre visible leur population et se mêlent de l'identifier sous des angles sans cesse multipliés. L'appropriation et son cortège d'usages et de droits, la levée d'impôts et de recrues, l'établissement et le maintien d'un ordre public diversement décliné, la fourniture de corvées et de prestations et mille autres gestions et ingérences détaillent des tâches ancestrales et en créent de nouvelles. L'état-civil détaille les individus, le fisc détaille des richesses, le censeur surveille les mœurs, le juge connaît des litiges et ainsi de suite jusqu'au statisticien qui essaie de mesurer toutes choses et au sondeur qui s'inquiète des opinions. Toutes activités qu'aucun vocabulaire intemporel ne peut subsumer, tant leur très longue histoire s'est moulée de multiples façons. Bref, la société en fonctionnement tend à objectiver les éléments d'ordre $\mathbf{H}$ qu'elle contient.

Par et pour ce travail de construction-vérification, des métiers prennent forme peu à peu. Le soignant, l'enseignant, le notaire, le statisticien et dix mille autres professionnels d'aujourd'hui ont émergé, au fil des millénaires, de précédents variés. Souvent encore, des prêtres, très divers figurent dans ce répertoire. D'autres lignées se développent hors l'emprise directe d'un Etat ou d'une confession, pour les besoins du commerce, de l'industrie, du spectacle, etc. Plus tardivement, la multi-médiatisation façonne d'autres spécialités, tandis que se multiplient les soins psychologiques et autres d'échelle individuelle, sans faire disparaître pour autant les bateleurs, rebouteux et cartomanciennes de vieille tradition A la différence des travaux paysans, artisanaux et industriels qui convertissent des éléments d'ordre $\mathbf{N}$ en produits finis, tous ces métiers s'activent quant à eux sur des éléments d'ordre $\mathbf{H}$ pour leur fournir finalement des prestations "immatérielles". Disant cela, je pose une distinction trop tranchée, car les travaux "matériels" et les prestations "immatérielles" s'entremêlent de plus en plus intimement dans l'aire des entreprises, sinon dans celle des administrations. Mais cela me permet de souligner une tendance forte du systè- 
me mondial actuel qui est d'approfondir considérablement l'bjectivation de l'ordre $\mathbf{H}$, car les métiers qui s'activent à cette échelle contribuent de plus en plus massivement au travail de constructionvérification de cet ordre.

\section{Du savoir à l'ignorance, allers et retours.}

Bref les objets $\mathbf{S}$ et $\mathbf{H}$ acquièrent une certaine solidité qui les rend accessibles à des sciences aussi spécialisables que les sciences naturelles, sinon dotées d'équipements en tous points semblables. Ce décalage qui est résorbable pour ce qui est des outils façonnés par les philosophies et pour les "expériences de pensée", devient moins maîtrisable quand il s'agit de toucher au "vivant", au "sensible", en s'approchant "par trop" des individus de l'espèce animale-humaine. Je ne développe pas cette problématique, car cela m'entraînerait dans l'énorme sophistique du "sujet" laquelle est profondément enracinée dans tous les coutumiers de la vie quotidienne et produit d'incessantes floraisons, bien arrosées par la plupart des appareils idéologiques et enrichies de pousses nouvelles quand quelque novation du savoir dérange les idées reçues.

Ces résistances et leurs regains ne doivent pas surprendre, elles tiennent aux fragilités naturelles de l'homme pensant et mortel et non pas aux insuffisances de l'instruction publique ou des "vulgarisations scientifiques", même si ces facteurs les accroissent assurément. En effet, quand il déborde des savoir-faire aux résultats patents, l'homme pensant et mortel ne peut s'oublier que par la contrainte de théories et de méthodologies soumises aux validations de collectivités organisées. Non que l'unanimité s'établisse jamais dans ces savantes institutions, mais bien parce que leurs débats font partie des contrôles publics, seuls vérificateurs substituables à l'évidence du produit matériel. La science est ce qui vérifie - c'est-à-dire rend vrai - le dire se prétendant savoir. Non vérifié de la sorte, un prétendu savoir n'est pas, pour autant, une sottise. Il est repris - ou non - d'autre manière et dans un 
autre contexte : l'histoire de toutes les disciplines scientifiques ou aspirant à la scientificité est pleine de telles exhumations. Ceci d'autant plus qu'aucun savoir ne peut être porté à l'absolu : la vérité est toujours conditionnelle, elle vaut jusqu'à preuve du contraire, elle n'épuise pas le trésor de l'ignorance savante.

En pratique, l'important est donc d'échafauder toujours plus de moyens de construction-vérification pour pénétrer toujours mieux les objets $\mathbf{S}$ et $\mathbf{H}$, sans oublier l'objet $\mathbf{N}$. Dans ce travail, le matériau qui s'est révélé le plus utile à l'expérience s'appelle, selon les convenances de chaque époque, chiffre, modèle, mathématique, statistique, etc. Pour bien gâcher ce ciment de la pensée, il faut veiller à la taille des pierres et des briques qu'il assemble. Je répète cette phrase en l'élucidant: il faut veiller à la taille (découpe, repérage, espace-temps à considérer, etc) des pierres (données dûment conçues) et des briques (statistiques, enquêtes, etc.) qu'il assemble (modèles, théories, etc.). Mais l'échafaudage porteur et la bâtisse achevée ne sont pas tout : encore faut-il faire une bonne part du chemin qui sépare ce savoir neuf du tout venant des humains, ce qui suppose un savant effort de vulgarisation, par l'intermédiaire de relais pertinents. Car rien n'est pire qu'une "communication savante" donnée en pâture à des quasiignorants, fussent-ils armés de medias puissants : c'est ouvrir le panthéon des sottises, des à-peu-près, des "réfutations" ineptes, des relances de vieilles erreurs, etc.

À preuve ? le sort réservé au tome 6 de ma Société.

\section{Robert FOSSAERT}

Décembre 2009

\section{Fin du texte}

